\title{
DRIVING MODEL OF BIG DATA GOVERNANCE IN URBAN COMMUNITY SAFETY SERVICE
}

\author{
Zhao-Ge Liu and Xiang-Yang Li \\ School of Management, Harbin Institute of Technology \\ Room 601, No. 13, Fayuan Street, Nangang District, Harbin, 150001, China
}

\begin{abstract}
Community is the basic unit of public safety management. A good community safety service is the key of improving community safety level. At the era of data explosion, big data based decision making has been widely adopted to facilitate community safety service. To achieve big data sharing and service value increasing, big data governance is the prime mode. Current big data governance studies lack an integrated model for comprehensively solving different data sharing problems. Models have dual advantages including supporting organizational synergy and supporting resource reuse. This paper puts forward a kind of driving model of big data governance in urban community safety service, integrating scenario-based driven, data-based driven and model-based driven approaches. The scenario-driven part of driving model provides the overall solutions for big data governance. The data-based driven level provides big data governance with objective evidence. The model-based level provides effective operational modes to the problem solving in big data governance. Through a use case, the rationality and effectiveness of the driving model are verified.
\end{abstract}

\section{KEYWORDS}

Community, Public Safety, Safety Service, Big Data Governance, Driving Model

\section{INTRODUCTION}

Urban community safety can be understood from two perspectives, which are state and degree. From the first perspective, researchers define community safety as the state that the risks of incidents threatening community residents' safety can be controlled (Wei and Lovegrove, 2013; Elbasyouny and Elbassiouni, 2013). From the second perspective, community safety is similar to community safety risk, which refers to the stable degree of external environment, including the community living conditions, community management mechanism and public service capacity. Safety service can be understood as all kinds of measures applied to guarantee safety. At the background of data explosion, it is concerned by both academia and industry that how to realize the mechanism innovation of community safety service by integrating, sharing and using the big data resources in community safety management. To solve this important problem, big data governance becomes the prime mode.

As a part of generalized information governance plan, big data governance is to make policies including data optimizing, privacy protecting and data realization relevant to big data. Big data governance framework includes three dimensions that are big data categories, big data governance principles and industries and functions of big data governance (Soares, 2014). Division of big data categories can help solve relevant problems such as data tracing, data right and data processing (George et al., 2016). Big data governance principles can be understood as major problems which need to be solved in big data governance. Tradition information governance principles such as organization, meta-data management, safety and privacy, data quality, business process integration, master data integration and information life cycle management can also be used in big data governance (Liu et al., 2016). Big data analysis is driven by specific scenes which contain certain industries and functions. Big data analysis can be applied to various industries and functions such as marketing, risk management, customer service, data sharing, information technology and human resource management (Martin et al., 2017). 
To sum up, existing studies of big data governance have basically established the general framework of big data governance and provide simple solutions to common problems in big data sharing. At different fields using big data based decision making methods, a lot of advanced technical achievements has emerged. However, existing studies are scattered and incomplete in the solving of complex governance problems. The solving process of integrative problems lack systematization. Specially, most of big data sharing and governance problems involve problems of different levels such as regulation, mater data and meta-data. It's difficult to solve the problems effectively only using single governance methods. For example, the data privacy and security problem involves problems including the regulation in organizational level and data structure in meta-data level.

Model driven decision making activities have dual advantages including supporting organizational synergy and the reuse of knowledge resource. Specifically, at the background of complex system, models can establish the relationship among various work or activities. Model driven method has been applied to many fields, and a lot of advanced methods have been proposed (Hidayet and Geylani, 2014). This paper proposes a kind of driving model of big data governance with certain degree of universality and hierarchy, which includes three levels that are scenario-driven level, data-driven level and model-driven level, separately realizing solution generating, data analyzing and process supporting.

\section{THE BASIC CONTENTS OF BIG DATA GOVERNANCE IN URBAN COMMUNITY SAFETY SERVICE}

According to the framework of big data governance and surveys on successful big data organizations (Hidayet, and Geylani, 2014), this paper generalizes the problems of big data governance in urban community safety service into three aspects that are data demand-supply, data sharing and data processing, coming with the basic contents of big data governance that are data demand-supply planning, data sharing strategy planning and multi-source data processing.

Data demand-supply planning, DDSP. Data demand-supply problem is pretty complex, involving specific fields such as data right, data lifecycle and data transaction. Data demand-supply planning is to make improvement projects according to the factors causing complexity to guarantee that the demand-supply plans are reasonable, rational and legal. Data demand-supply plans are designed according to three indicators that are reasonable data demands, rational choosing of data supply and legal data transfer. Among them, Reasonable data demands mean that all the specific requirements for data demands can be basically realized with regulation and techniques. For example, the time and space ranges of sharing data should be reasonable, or the data supply organizations may not be willing to supply the data. Rational choosing of data supply parties refers to choosing the data supply parties with acceptable data quality and data cost. For example, investigate and test the data quality of supply parties, and decide whether regulating supply parties or choosing other supply parties according to test results. Legal data transfer means that the processes of data supply of supply parties, data receiving of demanding parties and the data sharing among the different parties need to be consistent with existing laws and data right claims, in order to effectively support the activities of relevant stakeholders.

Data sharing strategy planning, DSSP. In terms of specific data demand-supply plan/network, data sharing strategy planning is to design strategies to make big data sharing extent meets the demands of data demanding parties. DSSP involves the contents of two levels that are organization and technique. At the level of organization, DSSP aims to design data sharing strategies through the games between demand and supply parties. At the level of technique, SEA pays more attention to the problem that how to facilitate data sharing with secure and reliable technical approaches.

Multi-source data processing, MSDP. Multi-source data processing is to solve the data processing problems at technical level. Specifically, MSDP includes multisource data processing, heterogeneous data processing and mixed conditions. MSDP takes the basic mode of data integration, which includes 4 parts that are pattern alignment, entity linking, conflict resolution and relationship inferring. Pattern alignment is used to find the actual same data with different semantic expression. For example, different governments often don not have the same description of community feature data. Entity linking technique is used to provide a similarity calculation method for discovering the actual same entity with different features, which is the key of big data integration. The key of entity linking technique lies on finding similar entities and processing. 
Conflict resolution technique is applied to reduce the problems including mode heterogeneity, semantic conflict and data redundancy produced in the process of big data integration and fusion. The conflict resolution technique is corresponded with pattern/ontology alignment technique, but pays more attention to the conflict problems in big data integration. Relationship inferring technique infers possible relationship to create value for achieving system functions using the techniques above.

\section{DRIVING MODEL CONSTRUCTION OF BIG DATA GOVERNANCE IN URBAN COMMUNITY SAFETY SERVICE}

According to the contents of big data governance, a kind of driving model of big data governance is proposed, as shown in Figure. 1. In the solving of big data governance problems, descriptive information, process data, and relevant models are all important. Descriptive information can help structure complex problems and express them, and further drive the overall strategy planning. Process data analysis can provide objective evidence for decision making. Process models provide a series of standard procedures of governance problem solving. Effectively using models can improve efficiency and effectiveness. Integrating the three types of resources, the driving model of big data governance includes three levels that are scenario-driven level, data-driven level and model-driven level. The hierarchical driving model can integrate scenario-driven, data-driven and model-driven methods, separately realizing solution generating, data analyzing and process supporting.

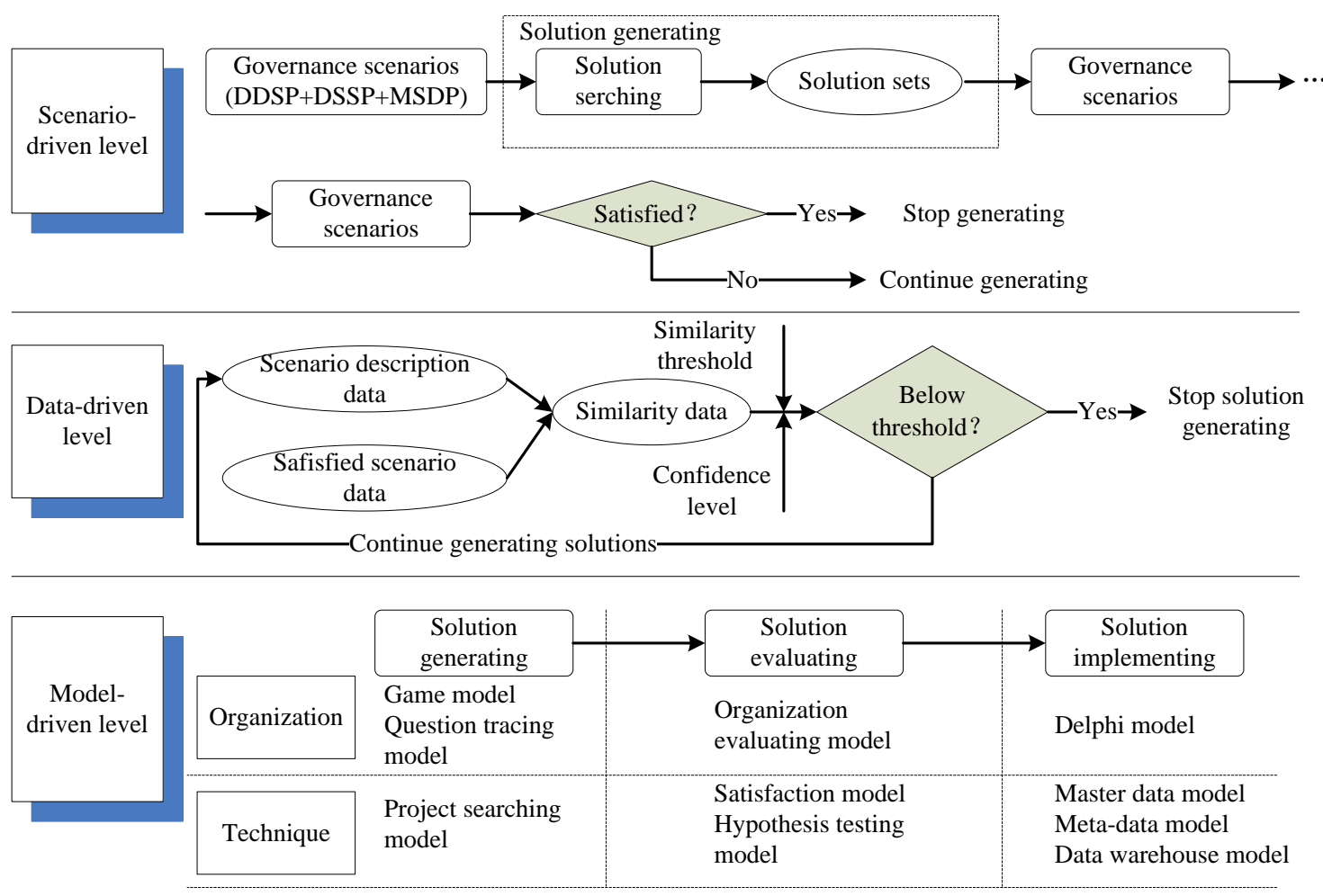

Figure 1. Driving model of big data governance in urban community safety service

Scenario-driven level, SDL. In big data governance, governance scenario refers to all kinds of problem description information. The major problems existing in current big data governance can thus be obtained by scenario analyzing. In scenario-driven level, the overall solving solutions are provided. The structured integration of related information gives a clear problem description, and the solution searching module intellectually generates guiding solutions. Via the continuous problem description and problem solving, the original problems are solved step by step. For example, in terms of the multi-source data processing problem, data integration techniques are identified that can effectively solve it. As a result, the data integration 
techniques are considered as overall solution. The multi-source data processing problem is originally solved in the new governance scenarios, but data demand-supply and data sharing problems are still remained in the new governance scenarios. Consequently, solutions need to be continuously induced to solve the problems. Finally, an originally general plan is generated as the results of SDL, which actually includes a set of solutions.

Data-driven level, DDL. Data-driven level provides big data governance with the objective evidence to make decisions of problem solving. It supports decision making with the input data, process data and output data at each decision making point. In the generation of solutions, the similarity between real scenarios and satisfied scenarios can be calculated with Euclidean distance. Consequently, the intellectually decision making can be achieved based on giving similarity thresholds and confidence level.

Model-driven level, MDL. Model-driven level provides big data governance with effective and standard procedures of the solving of big data governance problems. It realizes the generating, evaluating and implementing of solutions by calling and using relevant algorithms. For example, in terms of data sharing strategies in solution generating processes, the stakes of all stakeholders need to be coordinated. Further, the data sharing extent can be improved at the level of organization. In this process, the game model among multi-agents is used.

\section{CRUCIAL TECHNIQUES OF BIG DATA GOVERNANCE IN URBAN COMMUNITY SAFETY SERVICE}

The solutions of big data governance problems can be generated with the driving model of big data governance. In this part, the crucial techniques involved are discussed based on big data governance driving model. In fact, big data governance driving model is to gradually solve problems by scenario identifying and solution searching. Consequently, scenario identifying and solution searching become the most important parts in the driving model. Among them, scenario identifying is to figure out the problems, which is realized by using field knowledge. The knowledge here includes the experience knowledge contained in historical cases, the structured knowledge contained in rules, universal recognition of people, and so on. In this section, we take examples of mess map and case-based reasoning to illustrate the technical theories of scenario identifying and solution searching.

\subsection{Scenario Identifying Technique and Mess Map}

Scenario identifying technique is used to reduce the uncertainty of current scenarios by continuously mining the reasons causing governance problems. Mess map is a kind of question tracing technique, which can help find the reasons and describing the scenarios. As a tool of dealing with conflict opinions and data, mess map can help stakeholders in complex problems to form unified opinions in terms of social messes, which refer to fuzzy social problems that don't have direct solutions. The basic thinking of mess map is that the problems can be solved if the sources of problems can be traced by tracing reasons from the results of problems. Mess map provides the integral framework of analyzing problems by visual expression forms. It helps find the solutions by cross-organization coordination and communication.

Specially, mess map includes a kind of question tracing algorithm, which is a kind of procedure level technique that drives the operation of mess map. The leading departments of question tracing jointly achieve problem solving by man-machine interaction, as shown in Figure. 2. In the figure, $\mathrm{U}_{\mathrm{ij}}$ refers to the uncertainty in the jth stage of question tracing in terms of question $\mathrm{i}$. If $\mathrm{U}_{\mathrm{ij}}=0$, the uncertainty is reduced completely, which means the solutions have been obtained.

\subsection{Experience Learning Technique and Case-Based Reasoning}

Experience learning technique is another kind of method to obtain solutions of problems, which solves problems by reusing historical experience. Case-based reasoning (simplified as CBR) is a typical kind of experience learning technique. The most obvious features of CBR are structuring experience and programming experience learning processes. It supports the intelligent generating of solutions and doesn't 
involve man-machine interaction, which improves the objectivity of experience learning. The key of CBR is similarity calculation of scenarios, it refers to extract experience embedded in cases to guide practice by sorting the cases that have similar governance scenarios. In the solution generating of big data governance, it's necessary to match the scenarios of problems between current and case scenarios. The calculation method of similarity is as follows.

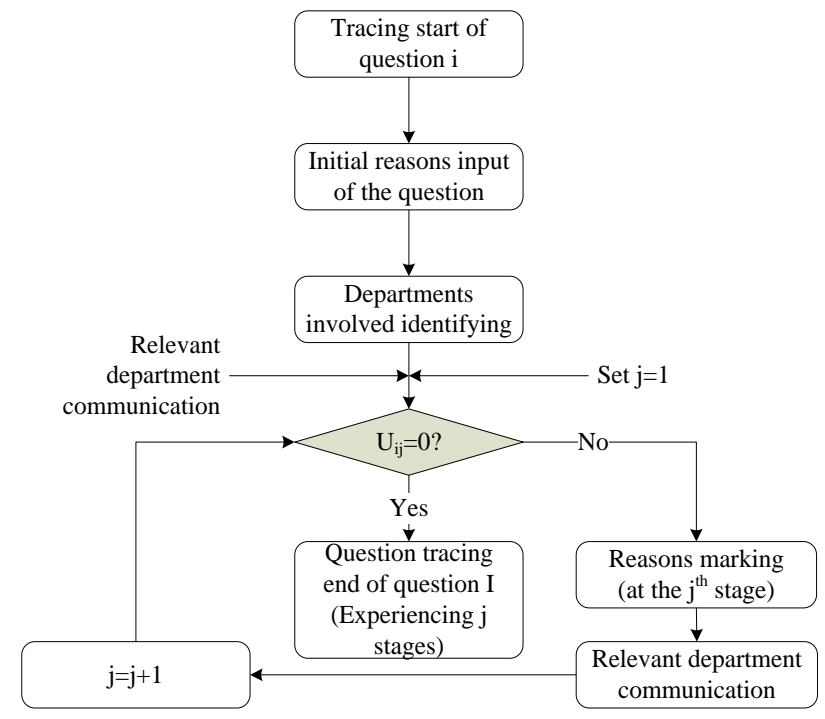

Figure 2. Question tracing alogorithm

Set $c a_{0}$ as real case, which means the case with a set of scenario features. Set $m e_{0 j}$ as the value of the real case in feature $j$. In CBR, the similarity is calculated through the calculation of Euclidean distance or Manhattan distance of two cases.

Distance of scenario features. $\forall i \in[1, m], \forall j \in[1, n]$, the distance between in scenario feature $j$ can be represented in (1).

$$
d i s_{0 i j}=\left|m e_{0 j}-m e_{i j}\right|
$$

Distance of cases. $\forall i \in[1, m]$, set $d i s_{0 i}$ as the distance of two cases, it is calculated by integrating all of the distances of scenario features between the two cases, which can be represented in (2).

$$
d i s_{0 i}=\left(\sum_{j=1}^{n}\left(w_{j} \times d i s_{0 i j}\right)^{p}\right)^{\frac{1}{p}}
$$

Among them, $p$ denotes the norm. If $p=1$, the $d i s_{0 i}$ represents the Euclidean distance. If $p=2$, the $d i s_{0 i}$ represents the Manhattan distance. $w_{j}$ denotes the weight of scenario feature $j$, and $\sum w_{j}=1$.

Similarity of cases. The similarity $L I K_{0 i}$ between two cases can be converted from the distance of the cases, which can be represented in (3).

$$
L I K_{0 i}=\frac{1}{1+\alpha \times d i s_{0 i}} \quad(i=1,2, \mathrm{~L}, m)
$$

Among them, $\alpha \in[0,1]$ is the similarity coefficient. Generally, the value is set as 1 .

It is worth to note that the case here can be divided as local cases and external cases according to case sources. Cases coming from different places have different applicability. Practice surveys need to be carried on to decide whether adopting the experience or not. 


\section{USE CASE}

In this section, a use case of big data governance in $\mathrm{S}$ city in China is illustrated to verify the rationality and effectiveness the proposed driving model. Specifically, the technique of solution searching here is CBR. In this section, we take position data contained in social media data as example, to realized the current governance scenarios and generate corresponding solutions. According to spot surveys, the scenario is generalized into 18 detailed types, as shown Table 1.

Table 1. Scenario feature of big data governance in the use case

\begin{tabular}{llll}
\hline Scenario feature type & Scenario feature & Feature value & Feature weight \\
\hline Governance problems & Data precision & 0.65 & 0.25 \\
& Data precision threshold & 0.85 & 0.20 \\
& Data precision state & Error (0) & 0.20 \\
& Master data & Social media text (1) & 0.20 \\
& Meta-data & Position (1) & 0.15 \\
& Whether authorized or not & Not (0) & 0.35 \\
& Protected data & Name (0) & 0.30 \\
& Protection technique & Access control (1) & 0.15 \\
& Privacy risk & 0.52 & 0.12 \\
& Risk threshold & 0.75 & 0.08 \\
Governance solutions & Level number of Access authority & 5 & 0.40 \\
& Access control regulation & No (0) & 0.30 \\
& Technical security level & 5 & 0.30 \\
Governance environment & Big data management department & Yes (1) & 0.28 \\
& Maturity of big data governance & $2^{\text {nd }}$ level (2) & 0.24 \\
& Big data management platform & Yes (1) & 0.21 \\
& Level number of big data governance & 3 & 0.15 \\
& Scores of techniques & 0.61 & 0.12 \\
\hline
\end{tabular}

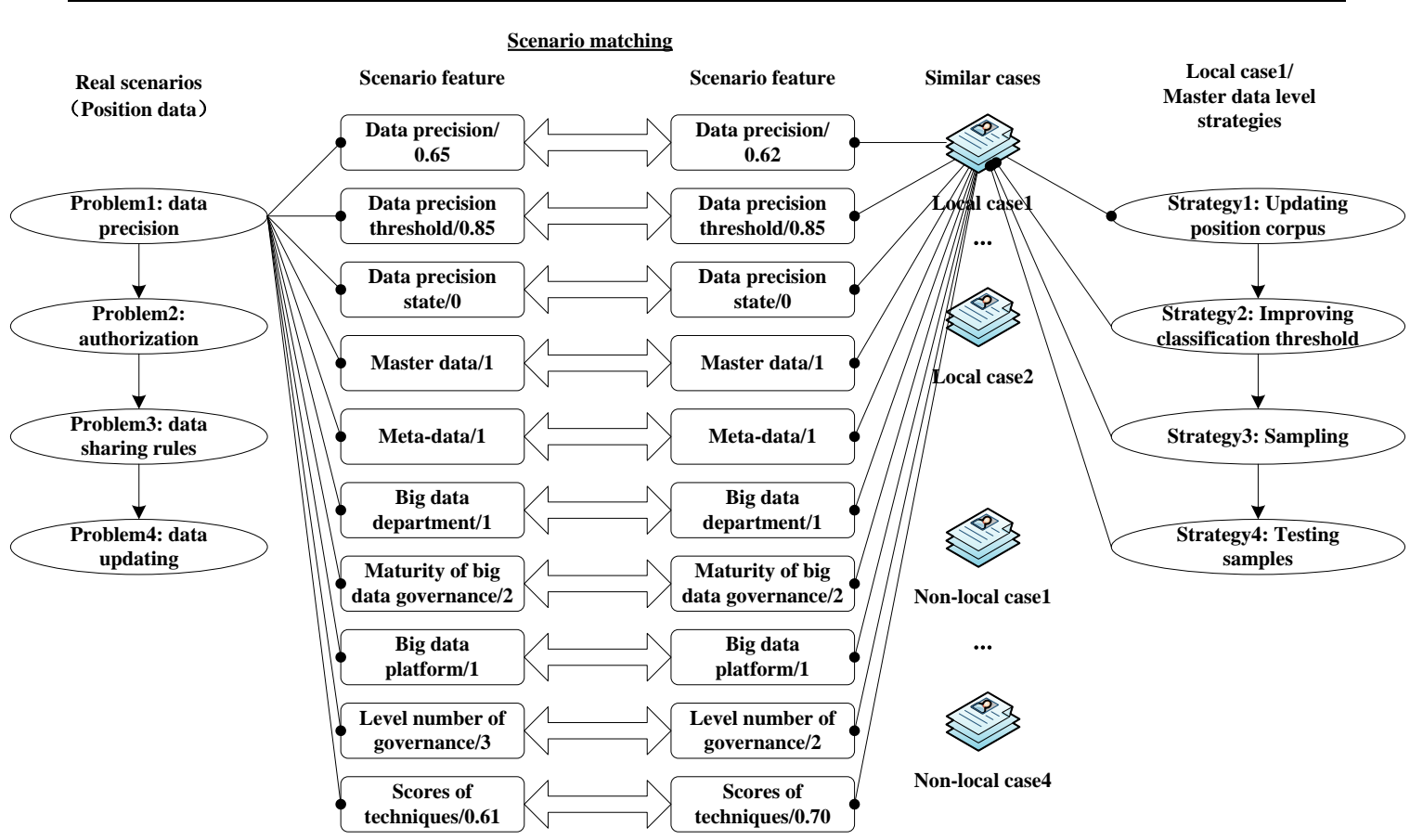

Figure 3. Scenario matching and solution genrating processes of big data governance in use case 
Using CBR technique to search for solutions of big data governance. According to eq(1) to eq(3), the similarity degrees between current scenarios and case scenarios can be obtained. The scenario matching process is shown in Figure. 3. Finally, specific strategies of big data governance are extracted from historical cases.

\section{CONCLUSION}

At the background of data explosion, big data governance has become the prime model to integrate, share and use the big data resources in community safety service, and further realize the mode innovation of services. Most big data governance problems involve various problems of different levels including regulation, master data and meta-data. In terms of this field problem, this paper puts forward a kind of driving model of big data governance with certain universality and hierarchy. The main contributions are as follows. Firstly, the basic contents of big data governance are extracted, including three kinds of contents that are data demand-supply planning, data sharing strategy planning and multi-source data processing. Secondly, the driving model of big data governance is proposed, including three levels that are scenario-driven level, data-driven level and model-driven level, separately realizing solution generating, data analyzing and process supporting. Thirdly, the critical techniques of big data governance driving in urban community safety service are discussed. Mess map and case-based reasoning are taken as examples to illustrate the technique theories of scenario identifying and solution searching. Of course, there are spaces which need further exploration, mainly reflecting as the connection of big data governance driving model with other models such as scenario models and reference models, and the empirical test of the driving model.

\section{ACKNOWLEDGEMENT}

This work is supported by the Major Research Project of Nation Natural Science Foundation of China named "Big data Driven Management and Decision-making Research" (No. 91746207), the General Program of Nation Natural Science Foundation of China (No. 71774043) and the Emergency Management Major Research Project of Nation Natural Science Foundation of China (No. 91024028).

\section{REFERENCES}

Elbasyouny, K., Elbassiouni, M. Y., 2013. Modeling and Analyzing Traffic Safety Perceptions: An Application to the Speed Limit Reduction Pilot Project in Edmonton, Alberta. In Accident Analysis and Prevention, Vol 51, pp 156-167.

George, G. et al, 2016. Big Data and Data Science Methods for Management Research. In Academy of Management Journal, Vol 59, pp 1493-1507.

Hidayet, B. S., Geylani, K., 2014. A Model Driven Architecture for the Development of Smart Card Software. In Computer Languages Systems \&Structures, Vol. 40, No. 2, pp 53-72.

Liu, X. et al, 2016. A Structured Analysis of Unstructured Big Data by Leveraging Cloud Computing. In Marketing Science, Vol 35, pp 363-388.

Martin, K. D. et al, 2017. Data Privacy: Effects on Customer and Firm Performance. In Journal of Marketing, Vol 81, pp 36-58.

Soares, S., 2014. Data Governance Tools: Evaluation Criteria, Big Data Governance, and Alignment with Enterprise Data Management. MC Press, Canada.

Wei, F., Lovegrove, G., 2013. An Empirical Tool to Evaluate the Safety of Cyclists: Community Based, Macro-level Collision Prediction Models Using Negative Binomial Regression. In Accident Analysis and Prevention, Vol 61, pp 129-137. 